\title{
SOLUTIONS OF LINEAR DIFFERENTIAL EQUATIONS \\ IN FUNCTION FIELDS OF ONE VARIABLE
}

\author{
MICHAEL F. SINGER
}

\begin{abstract}
ABSTRACr. Formal power series techniques are used to investigate the algebraic relationships between a function satisfying a linear differential equation and its derivatives. We are able to derive some conclusions, among them that an elliptic function satisfies no linear differential equation over a liouvillian extension of the complex numbers.
\end{abstract}

In [3], Rosenlicht noticed that if an element $y$ belonged to a liouvillian extension of a differential field, then the zeroes and poles of it and its derivatives must satisfy certain relations. His main tool was

TheOREM. Let $K$ be a field of characteristic zero, $k$ a subfield of $K, P$ a real discrete $k$-place of $K$ whose residue field is algebraic over $k, D$ a derivation of $K$ that is continuous in the topology of $P$ and that maps $k$ into itself. Let $x, y$ be nonzero elements of $K$ such that each of $x(P), y(P)$ is either 0 or $\infty$. Then:

(1) If $\operatorname{ord}_{P}(D x / x) \geqq 0$, then $\operatorname{ord}_{P}(D y / y) \geqq 0$. Here $D$ induces a derivation on the residue field of $P$. Denoting this residue field derivation by the same symbol $D$, for any $z$ in $K$ such that $\operatorname{ord}_{P} z \geqq 0$, we have $(D z)(P)=D(z(P))$.

(2) If $\operatorname{ord}_{P}(D x / x)<0$, then $\operatorname{ord}_{P}(D x / x)=\operatorname{ord}_{P}(D y / y)$ and, therefore, $\operatorname{ord}_{P}(y / x)=\operatorname{ord}_{P}(D y / D x)$. In addition, $(y / x)(P)=(D y / D x)(P)$.

Using this fact, he was able to show that certain differential equations have no liouvillian solutions. In this paper, we will show that the poles and zeroes of a solution of a linear differential equation and its derivatives must satisfy certain relations. With this we are able to mimic Rosenlicht's results and show that solutions of a large class of differential equations satisfy no linear differential equation (Corollaries 1 and 2). We will also prove a strengthened version of results of C. L. Siegel [5, p. 60] and L. Goldman [1, Corollary 3] and give an easy proof of a structure theorem of L. Goldman [1, Corollary 4].

The main tool of this paper is

Lemma. Let $k \subset K$ be differential fields of characteristic 0 . Let $w \in K$ satisfy the linear differential equation

$$
w^{(n)}-A_{n-1} w^{(n-1)}-\cdots-A_{0} w=B
$$

with the $A_{i}, B$ in $k$. Let $P$ be a discrete $k$-place of $k\langle w\rangle$ such that the derivation ' is continuous in the topology of this place. Then $\operatorname{ord}_{P} w<0$ implies that $\operatorname{ord}_{P}\left(w^{\prime} / w\right) \geqq 0$.

Received by the editors January 9, 1974 and, in revised form, December 5, 1974.

AMS (MOS) subject classifications (1970). Primary 12H05. 
Proof. Assume not; then $\operatorname{ord}_{P}\left(w^{\prime} / w\right)<0$ and so $\operatorname{ord}_{P} w^{\prime}<0$. Case 2 of the theorem now applies. We can conclude that $\operatorname{ord}_{P}\left(w^{\prime \prime} / w^{\prime}\right)=\operatorname{ord}_{P}\left(w^{\prime} / w\right)$ $<0$ and $\operatorname{ord}_{P} w^{\prime \prime}<0$. Similarly $\operatorname{ord}_{P}\left(w^{(k)} / w^{(k-1)}\right)<0$ and $\operatorname{ord}_{P} w^{(k)}<0$, and, in particular, ord ${ }_{P}\left(w^{(n)} / w^{(n-1)}\right)<0$. Using our linear equation, we have

$$
w^{(n)} / w^{(n-1)}=B / w^{(n-1)}+A_{n-1}+A_{n-2} w^{(n-2)} / w^{(n-1)}+\cdots+A_{0} w / w^{(n-1)} .
$$

I claim that the right-hand side of this equation has order 0 , which would give us a contradiction, and thus prove the lemma. First note that since

$$
\operatorname{ord}_{P}\left(w^{(n-2)} / w^{(n-1)}\right)>0 \text { and } \operatorname{ord}_{P}\left(w^{(n-3)} / w^{(n-2)}\right)>0,
$$

we have $\operatorname{ord}_{P}\left(w^{(n-3)} / w^{(n-1)}\right)>0$. Continuing in this way, we see that $\operatorname{ord}_{P}\left(w^{(n-i)} / w^{(n-1)}\right)>0$ for $2 \leqq i \leqq n-1$. Also since $\operatorname{ord}_{P} w^{(n-1)}<0$, $\operatorname{ord}_{P}\left(B / w^{(n-1)}\right)>0$. Therefore, the order of the right-hand side of the above equation is 0 .

COROllary 1. Let $k \subset K$ be differential fields of characteristic 0 and $y \in K$. Let $f$ be a polynomial in several variables over $k$ of total degree less than $n$, some positive integer, and $y^{n}=f\left(y, y^{\prime}, y^{\prime \prime}, \ldots\right)$. Assume further that the transcendence degree of $k\langle y\rangle$ over $k$ is 1 . Then $y$ satisfies no linear differential equation with coefficients in $k$.

Proof. Note that the transcendence degree assumption allows us to assume that the derivation is continuous in the topology of every $k$-plane [4, Lemma 1]. Assume that $y$ did satisfy such an equation. By the lemma, we would then have $\operatorname{ord}_{P}\left(y^{\prime} / y\right) \geqq 0$, where $P$ is a pole of $y$. This, in turn, implies that $\operatorname{ord}_{P} y^{(m)} \geqq \min \left(0, \operatorname{ord}_{P} y\right)$ for all $m$. Since $\operatorname{ord}_{P} y<0$, we have

$$
\left.\operatorname{ord}_{P} f\left(y, y^{\prime}, y^{\prime \prime}, \ldots\right) \geqq(n-1) \operatorname{ord}_{P} y>n\left(\operatorname{ord}_{P} y\right)\right)=\operatorname{ord}_{P} y^{n},
$$

which is a contradiction.

Corollary 2. An elliptic function satisfies no linear differential equation with coefficients in a liouvillian extension of the complex numbers.

Proof. Let $k$ be a liouvillian extension of the complex numbers and $y$ an elliptic function. Since $y$ satisfies the differential equation $\left(y^{\prime}\right)^{2}=y^{3}+a y$ $+b$, for some $a, b \in \mathbf{C}, a^{3} / 27+b^{2} / 4 \neq 0$, we could apply Corollary 1 , once we know that the transcendence degree of $k\langle y\rangle$ over $k$ is 1 . By looking at the above differential equation, we know it is at most 1 . If it were less, then $y$ would lie in a liouvillian extension of the complex numbers, contradicting the results on p. 372 of [3].

A homogeneous linear differential polynomial $L(W)$, with coefficients in $k$, is said to be linearly reducible over $k$ if there exist homogeneous linear differential polynomials $M(W), N(W)$, each of positive order, with coefficients in $k$, such that $L(W)=M(N(W))$. If $L(W)$ is not linearly reducible over $k$, it is said to be irreducible over $k$. We will need the following fact relating the reducibility of $L(W)$ to the behavior of its solutions under isomorphisms. Let $U$ be a universal extension of $k$ with constant field $C[2, \mathrm{p}$. 133], and $x$ a nonzero element of $U$ such that $L(x)=0$. Let $S$ be the set of differential $k$-isomorphisms of $k\langle x\rangle$ into $U, r$ the dimension of $T$, the $C$-span of 
$\{\sigma x \mid \sigma \in S\}$ over $C$, and $n$ the order of $L(W)$. I claim that there exist homogeneous linear differential polynomials $L_{n-r}(W)$ and $L_{r}(W)$, of order $n-r$ and $r$, with coefficients in $k$, such that $L(W)=L_{n-r}\left(L_{r}(W)\right)$.

To see this, we can assume that $r$ is less than $n$, and let $\sigma_{1} x, \sigma_{2} x, \ldots, \sigma_{r} x$ be a $C$-basis of $T$ and $L_{r}(W)=\operatorname{Wr}\left(W, \sigma_{1} x, \ldots, \sigma_{r} x\right) / \operatorname{Wr}\left(\sigma_{1} x, \ldots, \sigma_{r} x\right)$, where $\operatorname{Wr}\left(y_{1}, \ldots, y_{m}\right)$ is the Wronskian determinant. Any isomorphism of $k\left\langle\sigma_{1} x, \ldots, \sigma_{r} x\right\rangle$ into $U$ sends each $\sigma_{i} x$ into $T$ and so leaves the coefficients of $L_{r}(W)$ fixed. By the corollary on p. 388 of [2], the coefficients of $L_{r}(W)$ must be in $k$. Let $v_{1}=\sigma_{1} x, v_{2}=\sigma_{2} x, \ldots, v_{r}=\sigma_{r} x, v_{r+1}, \ldots, v_{n}$ be a fundamental system of solutions of $L(W)$ in $U$. Every differential $k$-isomorphism of $k\left\langle L_{r}\left(v_{r+1}\right), \ldots, L_{r}\left(v_{n}\right)\right\rangle$ into $U$ sends each $L\left(v_{r+i}\right)$ into the $C$-span of $L\left(v_{r+1}\right)$, $\ldots, L\left(v_{n}\right)$ and so leaves the coefficients of

$$
L_{n-r}(W)=\operatorname{Wr}\left(W, L\left(v_{r+1}\right), \ldots, L\left(v_{n}\right)\right) / \mathrm{Wr}\left(L\left(v_{r+1}\right), \ldots, L\left(v_{n}\right)\right)
$$

fixed. Therefore, $L_{n-r}(W)$ also has its coefficients in $k$. Since the coefficient of $W^{(n)}$ in both $L(W)$ and $L_{n-r}\left(L_{r}(W)\right)$ is $1, L(W)-L_{n-r}\left(L_{r}(W)\right)$ is a homogeneous linear differential polynomial of order less than $n$, with $n$ linearly independent solutions. Therefore $L(W)=L_{n-r}\left(L_{r}(W)\right)$. In particular, if $L(W)$ is irreducible it has a fundamental set of solutions of the form $x, \sigma_{1} x, \ldots, \sigma_{n-1} x$, where $x$ is any nonzero solution and the $\sigma_{i}$ 's are differential $k$-isomorphisms of $k\langle x\rangle$ into $U$.

COROllary 3. Let $k \subset K$ be differential fields of characteristic 0 and $w \in K$ which satisfies the linear differential equation $L(W)=B$, where $L(W)=W^{(n)}$ $-A_{n-1} W^{(n-1)}-\cdots-A_{0} W$ and the $A_{i}$ and $B$ are in $k$. If the transcendence degree of $k\langle w\rangle$ over $k$ equals 1 , then the homogeneous equation $L(W)=0$ has a solution $u$ such that $u^{\prime} / u$ is algebraic over $k$. If $L(W)$ is irreducible over $k$, then $L(W)=0$ has a fundamental set of solutions $u_{1}, \ldots, u_{n}$ such that each $u_{i}^{\prime} / u_{i}$ is algebraic over $k$.

Proof. The second assertion follows from the first and the remark at the end of the preceding paragraph.

To prove the first assertion, let $P$ be a pole of $w$. By the lemma, we have $\operatorname{ord}_{P}\left(w^{\prime} / w\right) \geqq 0$. Using case 1 of the Theorem, and observing that $w^{\prime}=w^{\prime} w / w$,

$$
\begin{aligned}
w^{\prime \prime} & =\left(\left(w^{\prime} / w\right)^{\prime}+\left(w^{\prime} / w\right)^{2}\right) w, \\
w^{\prime \prime \prime} & =\left(\left(w^{\prime} / w\right)^{\prime \prime}+3\left(w^{\prime} / w\right)^{\prime} w^{\prime} / w+\left(w^{\prime} / w\right)^{3}\right) w, \ldots, \\
w^{(n)} & =\left(\left(w^{\prime} / w\right)^{(n-1)}+n\left(w^{\prime} / w\right)^{n-2} w^{\prime} / w+\cdots+\left(w^{\prime} / w\right)^{n}\right) w,
\end{aligned}
$$

we see that $\left(w^{\prime} / w\right)(P)$ is an algebraic solution of the equation

$$
\begin{aligned}
V^{(n-1)}+n V^{(n-2)}+\cdots & +V^{n}-A_{1}\left(V^{(n-2)}+\cdots+V^{n-1}\right)-\cdots-A_{n} \\
& =(B / w)(P)=0 .
\end{aligned}
$$

We can now find a $u$ in some differential extension field of $k\left(\left(w^{\prime} / w\right)(P)\right)$ such that $u^{\prime} / u=\left(w^{\prime} / w\right)(P)$. This $u$ will then satisfy the homogeneous linear differential equation $L(W)=0$. 
Corollary 4. Let $k \subset K$ be differential fields of characteristic 0 and $z \in K$ a solution of a linear differential equation with coefficients in $k$. Assume that the transcendence degree of $k\langle z\rangle$ over $k$ is less than or equal to 1 . Letting $\bar{k}$ be the algebraic closure of $k$, we can then find $a v$ in $\bar{k}\langle z\rangle$ such that $z$ is algebraic over $k\langle v\rangle$ and $v$ satisfies a linear differential equation of order 1 over $\bar{k}$.

Proof. If the transcendence degree of $k\langle z\rangle$ over $k$ is zero, we are done. Assume $z$ is transcendental over $k$. Let $v$ be an element of $\bar{k}\langle z\rangle$, transcendental over $k$, which satisfies a linear differential equation over $\bar{k}$ of least order $r$, which we may assume is bigger than 1 . Let $L(V)=B$ be a linear differential equation of order $r$ that $v$ satisfies. By Corollary 3 we know that $L(V)=0$ has a solution $u$ such that $u^{\prime} / u$ is in $\bar{k}$. Letting $S$ be the set of differential $\bar{k}$-isomorphisms of $\bar{k}\langle u\rangle$ into the universal domain $U$, the dimension of the $C$ span of $\{\boldsymbol{\sigma} u \mid \sigma \in S\}$ is 1 . Using the paragraph preceding Corollary 3, we can conclude that $L(V)=L_{r-1}\left(L_{1}(V)\right)$, where $L_{r-1}(V)$ and $L_{1}(V)$ are homogeneous linear differential polynomials of order $r-1$ and 1 , with coefficients in $\bar{k} . L_{1}(v)$ is in $\bar{k}\langle z\rangle$ and satisfies $L_{r-1}(V)=B$, a linear differential equation of order less than $r$. Therefore, $L_{1}(v)$ must be in $\bar{k}$ and $v$ satisfies a linear differential equation of order 1 over $\bar{k}$, a contradiction. Therefore, $r=1$.

Both Corollary 4 and a weaker form of Corollary 3 were proven by L. Goldman [1] using the theory of differential polynomials and, in particular, the leading coefficient theorem of Ritt, which we have avoided.

\section{REFERENCES}

1. L. Goldman, Solutions of first order differential equations which are solutions of linear differential equations of higher order, Proc. Amer. Math. Soc. 10 (1959), 936-939. MR 22 \# 9489.

2. E. R. Kolchin, Differential algebra and algebraic groups, Academic Press, New York, 1973.

3. M. Rosenlicht, An analogue of l'Hospital's rule, Proc. Amer. Math. Soc. 37 (1973), 369-373. MR 47 \#666.

4. - On the explicit solvability of certain transcendental equations, Inst. Hautes Études Sci. Publ. Math. No. 36 (1969), 15-22. MR 41 \#3454.

5. C. L. Siegel, Transcendental numbers, Ann. of Math. Studies, no. 16, Princeton Univ. Press, Princeton, N. J., 1949. MR 11, 330.

Department of Mathematics, State University of New York, Stony Brook, New York 11794 